\title{
La apropiación de la temática de los derechos humanos por los regímenes marroquí y tunecino: retórica y realidad
}

\author{
Laura Feliu \\ Profesora de Relaciones Internacionales \\ Investigadora del Centre d'Estudis scbre la Pau i el Desarmament \\ Universitat Autònoma de Barcelona. 08193 Bellarerra (Barcelona). Spain
}

\section{Resumen}

La problemática de los derechos humanos emerge con fuerza a finales de la década de los ochenta en países como Marruecos y Túnez. Uno de los ámbitos en los cuales se manifiesta este protagonismo es en la apropiación de la temática por parte de los regimenes en el poder. En estos años se introduce con fuerza la cuestión en el discurso político; se implantan una serie de medidas dirigidas hacia la prevención de transgresiones del derecho y se crean instituciones que han de velar por una mejora de este campo. Paradójicamente, este proceso no conlleva un avance en el proceso democratizador ni en la situación de los derechos humanos a corto plazo y testimonia, en cambio, el valor que ha adquirido la temática como instrumento político.

Palabras clave: derechos humanos. Marruecos, Túnez, movimiento de derechos humanos, democracia.

Abstract. Rethinking the Mare Nostrum: the Mediterranean as an area for cooperatin and
security

The human rights issue springs tup at the end of the $80 \mathrm{~s}$ in countries like Morocco and Tunisia. One of the questions reflecting this prominence is its assumption by the groups in power. In the course of these years, human rights were clearly included in the political discourse; measures were approved for the prevention of legal transgressions; also a series of institurions devoted to the improvement in this sphere was created. Paradoxically this process does not mean progress in the democratization processes or in the observance of human rights in the short run but shows the value of human rights issue like a political tool.

Key words: human rights, Morocco, Tunisia, human rights movement, democracy.

\section{Sumario}

El discurso-compromiso sobre los derechos humanos

La institucionalización de los derechos humanos

Factores internos pata tuna comprensión de los cambios
La acción internacional en defensa de los derechos humanos

Evolución o revolución en el campo de los derechos humanos

Bibliografia 
No olvidemos que la moda de los derechos humanos ba abierto los ojos a la luz hace algunos años.

Hassan II, 1992: 182

Nuestro objetivo sigue siendo la garantía de la plenitud de los derechos bumanos.

Ben Ali, en Amnistía Internacional, 1994: 15

Los informes de diferentes organizaciones de derechos humanos nacionales e internacionales ponen en evidencia petiódicamente las deficiencias en la situación de los derechos humanos en los países del Magreb. En los casos de Marruecos y Túnez, la persistencia en las violaciones de los derechos humanos se ha visto acompañada, a finales de los años ochenta de un cambio de actitud de los regímenes en el poder en su aproximación a la temática. La cuestión de los derechos humanos parece haberse convertido en un magnífico instrumento de legitimación de la práctica política en unas coyunturas internas e internacionales especialmente delicadas.

Es básicamente en tres niveles en los cuales se manifiestan los cambios: en el discurso político oficial; en la creación de una uburocracia de derechos humanos" dependiente de las Jefaturas del Estado, y en la promulgación de una serie de reformas legislativas y adopción de gestos dirigidos, en principio, hacia una mejora de la situación de los derechos humanos.

\section{El discurso-compromiso sobre los derechos humanos}

El análisis del discurso político de los dirigentes marroquíes y tunecinos durante el período en el que se centra el presente artículo (1987-1994) pone de manifiesto su reciente transformación. El cambio va más allá de una mera renovación del vocabulario utilizado y evidencia una ruptura conceptual derivada de la paulatina transformación de la cultura política de las respectivas sociedades. El discurso político oficial de los dos paises magrebíes recoge algunos de los elementos que habían sido una constante en las reivindicaciones de la oposición y, sobre todo y unido a la anterior, del movimiento de derechos humanos. Esta apropiación de demandas responde básicamente a la voluntad de desarmar la crítica del contrario con sus mismas armas, canalizar las peticiones en la dirección deseada y, al mismo tiempo, fomentar cierta imagen de paternalismo omnipresente.

Dos son los conceptos que hacen su aparición con fuerza en el discurso de finales de los años ochenta: estado de derecho y derechos humanos. Ambos términos se encuentran presentes sólo esporádicamente en las proclamas anteriores y se observa una transformación notable de su significado desde aquellos primeros días de la independencia, a mediados de los años cincuenta. Trás esta evolución se encuentra sin duda la metamorfosis operada por un concepto 
clave, el de democracial. Si en un primer momento el térrmino se había concretado casi exclusivamente: en la reivindicación de una verdadera separación de poderes y en el reconocimiento de los componentes de una democracia formal - planteándose el modelo democrático como un objetivo a largo plazo-, a finales de los ochenta el concepto se aproxima más a un proyecto global de sociedad, en el cual la democracia ha de ser el medio para edificar una sociedad más igualitaria y justa. El perfeccionamiento jurídico del sistema y el respeto de los derechos humanos son piezas clave de esta nueva formulación de viejos términos.

El compromiso ineludible con la observancia de los derechos humanos y el imperio de la ley es afirmado repetidas veces en numerosos discursos de los últimos años. El Rey de Marruecos, Hasán $\mathrm{II}^{2}$, en su tradicional discurso del Trono de 1992 afirma que: «Los principios democráticos y los conceptos morales que Nosotros hemos evocado así como Nuestra fe en la preeminencia del Estado de derecho nos han llevado a otorgar a los derechos humanos un lugar preferente entre Nuestras preocupaciones" (Hassan II, 1993: 12).

Unos af́os antes el general Ben Alí, en su primer discurso frente a la nación tunecina en calidad de presidente, enumera lo que se ha dado a conocer como los "principios de noviembre" que han de guiar la construcción de una democracia «responsable» ${ }^{3}$. El objetivo último ha de ser la creación de: «una sociedad libre y democrática y un Estado pluralista que respete los derechos humanos [...] llevar a la práctica la noción de imperio de la ley, único medio de asegurar la prosperidad para los tunecinos en el contexto del respeto de los derechos humanos y de las libertades fundamentales" ${ }^{4}$. La referencia a los anteriores elementos es especialmente novedosa si consideramos su relegación explícita por el anterior presidente Habib Burguiba en la consecución de intereses consi-

1. Sobre el concepto de democracia en el Magreb central (Marruecos, Argelia y Túnez), véase el trabajo de Michel Camau (1971).

2. Hijo del sultán Muthammad V, accedió al trono de Marruecos en 1961. Bajo su mandato se edifica una democracia formal basada en un pluralismo controlado. Dos grandes grupos de parridos politicos son identificables: los "oficiališas", propiciados desde el régimen, y los integrantes de la oposición, herederos del núcleo inicial que formaba el Movimiento Nacional, que aglutinó diferentes tendencias políticas en stu lucha contra la metrópoli francesa antes de iniciar su progresiva fragmentación. En su confrontación con la monarquía para obtener el control del campo político-social, la oposición sufte una fuerte represión y tiene que aceptar la hegemonía casi absoluta de Palacio.

3. El acceso a la presidencia del general Zin El Abidin Ben Alí en noviembre de 1987, en aquellos momentos primer ministro, se produce a través de un golpe de estado umédicoconscitucionaly en el cual se invoca el arcículo 57 de la Constitución (que prevé la sustinución del presidente por el primer ministro cuando las condiciones de salud del primero le imposibilitan ejercer su cargo). De esra manera se pone fin al largo gobierno de Habib Burguiba, el "Combatiente Supremo", que había conducido el pais hacia la independencia; figura carismática identificada con la modernización del país, en los últimos años había gobernado valiéndose de métodos basados en una mezcolanza de autoritarismo y populismo.

4. Tercer informe presentado por Túnez al Comité de Derechos Humanos de Naciones Unidas el 12 de junio de 1989 (CCPR/c/52/Add. S, párrafo 2). 
derados prioritarios. Burguiba declaraba en 1963 que: «Nada puede obstaculizar la acción del Estado. Ninguno de los derechos Hamados derechos naturales del individuo ha de primar en cuanto se trata de la vida de la nación [...] Las libertades se han de proscribir si llevan prejuicio a la colectividad [...]" (Karem, 1991: 406).

\section{La institucionalización de los derechos humanos}

La alusión a la problemática de los derechos humanos va más allá de su introducción en el discurso oficial, al materializarse en una serie de instituciones y figuras competentes en el seguimiento del tema. La creación de una burocracia de derechos humanos pretende fundamentalmente canalizar las diferentes reivindicaciones presentes en el campo político-social y dar respuesta a una serie de críticas procedentes tanto del interior como del exterior del país. Es en Marruecos donde primero se inicia la edificación del complejo institucional ${ }^{5}$. Destacamos fundamentalmente dos instituciones.

La primera de ellas es el Consejo Consultivo de Derechos Humanos (CCDH), creado en abril de 1990 con una composición de 37 miembros escogidos por el monarca alauí entre los candidatos presentados por los principales partidos políticos, sindicatos y organizaciones de derechos humanos, entre otros colectivos, además de la presencia de una serie de figuras cercanas al régimen $^{6}$. El Consejo se perfila como un organismo que ha de asistir al monarca en todas las cuestiones relativas a los derechos humanos. En la práctica su acción se ha centrado básicamente en el análisis de aquellos puntos de la legislación que requieren una reforma mas urgente ${ }^{7}$. Su actividad, después de un primer año de interesantes iniciativas, será extremadamente irregular al depender estrechamente del impulso que le otorga el Rey.

Un segundo organismo de reciente creación es el Ministerio de Derechos Humanos, anunciado por Hasán II el 11 de noviembre de $1993^{8}$. Sus funciones son extraordinariamente amplias e incluyen todo tipo de atribuciones. Además de tareas generales relacionadas con la promoción del ideal de los

5. Sobre la burocracia marroqui de derechos humanos véase fundamentalmente Basri, Rousset y Vedel (1994).

6. De los convocados, únicamente la Asociación Marroquí de Derechos Humanos (AMDH) declinará la invitación a sumarse a la iniciativa, alegando la supeditación de la insticución a Palacio y la integración de personalidades conocidas por su desprecio hacia los derechos humanos.

7. Debemos llamar la atención sobre el hecho deque, según el funcionamiento lógico de toda democracia, correspondería al Parlamento el llevar a cabo una labor de propuesta de reformas legislativas relativas al establecimiento de un Estado de derecho.

8. Decreto núm. 2-94-33 del 24 de mayo de 1994 relativo a las atribuciones y a la organización del Ministerio de Derechos Humanos delegado delante del primer ministro (BORM 4262, 6/7/1994: 335-6). Un antecedente en la región es la creación en Argelia en julio de 1991 de un Minisrerio de Derechos Humanos presidido por Als Harún y que es reemplazado en febrero de 1992 por el Observatorio Nacional de Derechos Humanos (Martín Muñoz, 1993: 161). 
derechos humanos, se hace referencia a funciones de tipo legislativo y reglamentario y otras de difusión y propaganda. Dado el solapamiento con algunas de las funciones hasta ahora realizadas por el $\mathrm{CCDH}$, parece ser que el Ministerio se decantará primordialmente hacia una función educativa y de divulgación.

En el caso tunecino la institucionalización es especialmente completa, formando un entramado que difícilmente encuentra paralelo en páses del mundo occidental o de la esfera árabo-musulmana?.

En primer lugar se ha de destacar una institución paralela al CCDH marroquí, el Comité Superior de Derechos Humanos y de Libertades Fundamentales. Creado en abril de 1991 como un organismo dependiente de la figura del presidente, lo componen 27 miembros entre representantes de la administración (9), figuras independientes (10) y miembros de ONG y grupos relacionados con la defensa de los derechos humanos $(8)^{10}$. La función del Comité es aconsejar al presidente en relación con las reformas a emprender en este campo.

Un segundo grupo de instituciones de carácter no permanente son creadas en un contexto de especial crispación y represión. El presidente Ben Alí nombra en junio de 1991 una cornisión de investigación dirigida por Rachid Driss, antiguo embajador en las Naciones Unidas ${ }^{1 P}$. En vistas de la continuación de las transgresiones a la legalidad, en abril de 1992 Driss solicita al presidente la formación de una rueva comisión, que publicará sus resultados posteriormente.

Una tercera figura es el cargo de consejero particular del presidente en materia de derechos humanos, creado en junio de 1991. Además de aconsejar sobre la política presidencial, el Consejero realiza un seguimiento de las diferentes organizaciones de derechos humanos y recibe demandas de particulares ${ }^{12}$.

Por último destacamos la designación de un mediador de la administración con competencias relativas a los derechos humanos, en noviembre de $1992^{13}$. Este cargo, que ha de dar curso a las demandas de individuos sobre acciones públicas, se ha caracterizado por su inactividad.

9. Sobre la burocracia tunecina de derechos humanos véase Lawyers Committee (1993) y Amnistía Internacional (1994).

10. Decreto presidencial núm. $91-54$ del 7 de enero de 1991 (JOT 11/1/1991).

11. La Comisión se forma después que en mayo de 1991 murieran dos isiarnistas, presuntamente bajo torturas. La Liga Tunecina de Detechos Humanos (LTDH) había anunciado la creación de una comisión de investigación para seguir el asunto, mientras que Amnistía Internacional emitía días antes un comunicado especialmente duro. El informe final de la Comisión no llegará a publicarse. En una declaración conjunta en octubre de 1991, Ben Ali y Rachid Driss afirman que los abusos tienen un "carácter individual".

12. En un primer momento desempefia el cargo el profesor de Sociología de la Universidad de Túnez, ladh Ouederni, hasta su nombramiento como ministro de Justicia en junio de 1992, fecha en la cual es sustituido por el profesor de Detecho Internacional, Sadok Chaaba.

13. Decrevo 92-2143 de 1992. La petsona que desempeña el cargo es Hassin Cherif. 
A parte de estas instituciones centrales, otras iniciativas interesantes completarán el panorama de la institucionalización de la temática en los dos países ${ }^{14}$.

El compromiso verbal con la causa de los derechos humanos se completa, tanto en el caso de Marruecos como de Túnez, con dos tipos de gestos emanados desde la cúspide del poder. El primero de ellos son los cambios positivos efectuados en los principales dossiers relativos a los derechos humanos ${ }^{15}$. Estas acciones son especialmente interesantes, pero hay que relativizar su significancia, ya que no han comportado la inculpación de aquellas personas responsables de abusos y, especialmente en el caso de Túnez, las mejoras en ciertos ámbitos se han visto acompañadas de nuevos abusos o del reinicio de viejas prácticas. El segundo tipo de gestos es la promulgación de una serie de reformas que modifican los aspectos más deficitarios de la legislación. Su aplicación se mostrará extremadamente dificultosa por la falta de voluntad política para una completa puesta en práctica ${ }^{16}$.

\section{Factores internos para una comprensión de los cambios}

El objetivo que ha impulsado las transformaciones expuestas con anterioridad no parece ser la simple mejora de la situación de los derechos humanos. Esto se hace particularmente evidente en el caso de Túnez, donde el establecimiento de organismos que velen por los derechos humanos coincide con una agudización de la represión política ${ }^{17}$. ¿Qué motivos se encuentran pues detrás de tal mutación formal del campo de los derechos humanos? Sin pretender ser exhaustivos ni establecer una jerarquía entre los diferentes elementos apunta-

14. En el caso de Marruecos se crea en enero de 1991 una Dirección de Formación Personal, Codificación y Libertades Públicas en el Ministerio del Interior. En Túnez se insertan células de derechos humanos en prácticamente todos los Ministerios, se otorga anualmente una medalla de derechos humanos o se crean cátedras de derechos humanos en las facultades de Derecho. Estas previsiones han ido acompañadas, según fuentes oficiales, de medidas comunes a Marruecos y Túnez, como la dotación a los puestos de polića de Declaraciones Universales de los Derechos Humanos o los cursos sobre la matetia que reciben los miembros de las fuerzas de seguridad (Amnistfa Internacional, 1994: 9).

15. Paujatinamente son liberados los presos políticos marroqufes más conocidos, encarcelados desde los años setenta y pertenecientes en su mayoría al movimiento marxista-leninista; se promulga en julio de 1994 una amnistia de carácter prácticamente generał; en octubre de 1991 es derribado el terrible presidio de Tazmamart; en agosto de 1991 son puestos en libertad 269 saharauis que Marruecos había negado tener en su poder. En el caso de Túnez, con la llegada de Ben Alí a la presidencia, se liberan buena parte de los prisioneros polf́cicos (en 1987 unos 3000 miembros del partido islamista Movimiento de Tendencia Islámica, MTI, se encuentran en prisión) que superpueblan las cárceles en esos momentos.

16. Tanto en Marruecos como en Túnez las primeras leyes reformadas son las relativas al régimen de incomunicación (ley 67-90 del 30 de diciembre de 1991 y ley $87-70$ del 26 de noviembre de 1987, respectivamente) y a la prisión preventiva (Dahir núm. 1-91-110 del 30 de diciembre de 1991 en el caso de Marruecos).

17. El 1990 Amnistía Internacional cifra los prisioneros políticos en 8.000 , la LTDH contabiliza entre 2000 y 3000 y el movimiento islamista En-Nahda se tefiere a 30.000 (Daoud, 1993: 950). 
dos, ofrecemos en este apartado y en el siguiente algunos de los factores que han de ser tomados en cuenta al realizar una evaluación de la significación de las transformaciones observadas.

En primer lugar se ha de considerar el potencial legitimador inherente a la problemática de los derechos humanos, del cual los diferentes regímenes pretenden apropiarse. El avance en la formación del llamado "régimen internacional de derechos humanos» comporta la existencia de unas normas ampliamente aceptadas a nivel de Estados, aunque extremadamente limitadas a nivel de aplicación; este régimen adquirirá al final de la guerra fría un especial protagonismo en la agenda internacional ${ }^{18}$. ¿ंPor qué precisamente es en estos momentos cuando aparece la voluntad de apropiación de dicha legitimidad? El afianzamiento de los sistemas marroquí y tunecino se convierte en una necesidad acuciante en un momento en el cual las oposiciones políticas en ambos países suponen un peso considerable, el movimiento de derechos humanos se muestra especialmente activo y se apunta un cercano "despertar" de la sociedad civil. La temática de los derechos humanos pasa a ocupar un lugar prioritario en el intento de aproximación a la oposición política. En el caso de Marruecos ésta ha formado una coalición unitaria alrededor del Bloque Democrático (Kutla Dimoqratia), creado en mayo de 1992 después de múltiples intentos frustrados de actuación conjunta. De esta manera el panorama político marroquí se aproxima otra vez a la configuración "bipolar", de los primeros tiempos de la independencia, con sus extremos en el binomio monarquía-oposición (Leveau, 1993). Esta estructura se había diluido ocn la "domesticación" de las fuerzas políticas convertidas en una oposición legalista a costa de la práctica desaparición de aquellos sectores más radicales que propugnaban la revolución total. Las modificaciones del campo político coincidirán con el inicio de acciones concertadas en las cuales la reivindicación de respeto por los derechos humanos es la piedra angular.

En Túnez, el presidente Ben Alí se ve obligado a buscar apoyos que abarquen amplios sectores del espectro político y que ayuden a legitimar su repentino acceso al poder. Diferentes tendencias son integradas en el Pacto Nacional, formado en noviembre de 1988, que tiene como objetivo perfilar un «código de honor que ha de dictar las normas de conducta polírica, económica y social y promover la concertación" (Daoud, 1992: 778) ${ }^{19}$. Como en el caso marroquí, la temática de los derechos humanos es central para captar la confianza de sectores que ven con buenos ojos todo cambio respecto a las prácticas de la «era Burguiba».

18. Sobre el concẹto de régimen internacional y su aplicación al campo de los detechos humanos, véanse las obras de David P. Forsythe (1990), Jack Donnelly (1986 y 1989) o R.J. Vincent (1986a y 1986b).

19. Un pluralismo controlado se ha iniciado el 1988 a través de la promulgación de la ley núm. 88-32 de 1988 que permite la legalización de seis partidos además del gubernamental Agrupación Constitucional Democrática (RCD). El partido isłamista En-Nanda, dirigido pot R. Ghannuchi, es excluido de la competición política a través de la prohibición de todo partido político con una base confesional, étnica o regional. 
Juntamente con la actividad de la oposición, el movimiento de derechos humanos ha experimentado un extraordinario progreso en ambos países. En el caso de Marruecos esto se manifiesta tanto en la reactivación de dos organizaciones que se encontraban en un estado de aletargamiento a mediados de los ochenta, como en la creación de otras nuevas ${ }^{20}$. En el primer caso nos encontramos con la veterana Liga Marroquí de Defensa de los Derechos Humanos (LMDDH), creada en mayo de 1972 bajo el impulso de un grupo de militantes del Partido del Istiqlal (PI). Su relación con este partido comporta que su actividad sea elevada durante sus primeros cinco años de vida cuando el PI se encuentra fuera del gobierno-, para limitarse posteriormente a una crítica ponderada del régimen. Una segunda organización es la Asociación Marroquif de Derechos Humanos (AMDH), surgida en 1979 en un momento en el cual monarquía y fuerzas políticas se han acercado en un clima de consenso que tiene como telón de fondo la cuestión de la descolonización del Sahara Occidental ${ }^{21}$. La AMDH surgirá básicamente de la iniciativa de sectores de izquierda adscritos a la Unión Socialista de Fuerzas Populares (USFP), que a finales de la década se ha sumado a la causa común de la marroquinidad del Sáhara. Las desavenencias entre los partidarios de un diálogo estrecho con las autoridades centrales y las tendencias críticas provocan un desagradable incidente en 1983 que finaliza con la escisión del sector USFP-CAN (actual Partido de Avanguardia Democrática y Social, PADS), que arrastra consigo a la AMDH hacia una posición de marginalidad.

La actividad de la LMDDH y la AMDH se desarrolla a lo largo de unos años en los cuales la necesidad de "recuperar" el Sahara Occidental monopoliza la escena política y se convierte en el interés prioritario, en detrimento de una acción universal pro-derechos humanos ${ }^{22}$. Sin duda, el pacto implícito entre monarquía y oposición — que no se traduce en el final de la represión - se encuentra en el origen de la pasividad del movimiento de derechos humanos.

No es hasta los años 1987-1988 cuando el panorama se reactiva. En marzo de 1988 se constituye la Comisión de Coordinación entre la LMDDH y la AMDH que simboliza el relanzamiento de las dos organizaciones. El cambio coincide con la creación en diciembre de 1988 de una nueva asociación,

20. Sobre las organizaciones de derechos humanos marroqufes, véase Bennouna (1993a, 1993b), Karem (1991, 1993) y Basri, Rousset y Vedel (1994).

21. El territorio colonizado por Espafia pasa a ser administrado conjuntamente por Marrtecos y Mauritania después de la firma del Acuerdo de Madrid el 14 de noviembre de 1975. Diferentes resoluciones de las Naciones Unidas y de la OUA establecen la necesidad de celebración de un referéndum de autodeterminación del territorio -que el Frente Polisario reclama se convierta en un Estado independiente (la República Arabe Saharaui Democrática, RASD), frente a la voluntad anextonista de Marruecos. Este último país acepta formalmente la vá del referéndum en la cumbre de lá OUA de Nairobi en junio de 1981, al mismo tiempo que interpone numerosos obstáculos a su celebración.

22. El ejemplo más claro es la inexistencia de iniciativas relativas a la represion padecida por la población saharaui, tema tabú incluso hoy en dfa. 
la Organización Marroquí de Derechos Humanos (OMDH). La Organización pretende marcar un nuevo estilo de activismo independiente y alejado de los partidismos que han caracterizado las experiencias anteriores. Por este motivo, intenta inicialmente integrar en su seno las organizaciones de derechos humanos ya existentes; frente a la negativa de éstas, finalmente establecerá una politica de cuotas en la composición de los órganos directivos, la mitad de sus miembros han de ser figuras independientes y el resto representantes de partidos políticos. De esta manera se pretende asegurar la representación de sectores muy diferenciados de la vida política. A pesar de los esfuerzos realizados, la independencia frente a tendencias políticas concretas sigue siendo una asignatura pendiente ${ }^{23}$.

El movimiento tunecino de derechos humanos tiene unas peculiaridades que lo distinguen del anterior modelo. Una única organización representa el conjunto de la militancia pro-derechos humanos, lo que en un principio podría ofrecer una visión monocorde del movimiento. En realidad, la existencia aislada de la Liga Tunecina de Derechos Humanos (LTDH) esconde una actividad especialmente dirámica e integradora de tendencias mucho más dispares que en el caso marroquii ${ }^{24}$. La Liga se crea en 1977, un año después de la constitución de un núcleo inicial de quince personalidades de la izquierda, extrema izquierda y de unos pocos miembros del Partido Socialista Desturiano (PSD), con voluntad de hacer llegar al conjunto de la sociedad una serie de reivindicaciones. En aquellos momentos el PSD, que dirige en solitario el país ${ }^{25}$, intenta neutralizar la iniciativa con la presentación de una organización paralela y el otorgamiento de un único permiso de constitución. Obligados por las circunstancias, los pioneros del movimiento de derechos humanos optan por la creación de una organización unitaria que integre en su Comité Director siete desturianos de un total de 22 niembros. Estos constreñimientos iniciales contribuirán a que la organización aglutine elementos muy dispares, desde sectores comunistas a islamistas (estos últimos no presentes como tendencia en las organizaciones marroquíes) ${ }^{26}$, pasando por partidarios del régimen. Si en un principio se planteaban dudas sobre la independencia de la Liga respecto al gobierno, su decidida actuación a partir de 1985 contra la represión le otorga

23. En la organización se ha de destacar la preeminencia de los sectores ligados a la USFP. En el año 1994 tuvo lugar un interesinte debate en el interior de la Organización sobre la abolición del sistema de cuotas. Annque la propuesta no prosperó, se planteó la existencia de distorsiones provocadas por el hecho que ciertos militantes puedan en determinados momentos guiarse más por fidelidades hacia su partido que por consideraciones de interés general.

24. Sobre la LTDH véanse los trabajos de Mohamed Karem (1991, 1993).

25. El Partido Comunista había sido prohibido en 1963, lo que de hecho llevo a la instauración de un régimen de partido único, dirigido por el Neo-Destur, que un año más tarde se convierte en el PSD, pata transformarse con la presidencia Ben Alí en el actual RCD.

26. Los grupos islamistas han sufrido una persecución implacable en Túnez, especialmente desde 1981. Estos sectores han elaborado un interesante discurso relativo a los derechos humanos, más avanzado que sus homólogos magrebíes. 
una cierta reputación. En estas fechas se estructura la organización y se integran nuevos miembros procedentes en su mayor parte de la desmantelada Unión General de Trabajadores Tunecinos (UGTT). A partir de 1991 su crítica a la política de la presidencia Ben Alí y a la falta de progresos en materia de derechos humanos será mucho más acerada que en años anteriores y reflejará la decepción ante el incumplimiento de las promesas formuladas.

El panorama final del movimiento de derechos humanos en ambos paises magrebíes es especialmente fecundo ${ }^{27}$. Tanto en Marruecos como en Túnez las especiales características del campo político, con estrechos márgenes de maniobra y la existencia de límites muy marcados, habrán condicionado decisivamente la evolución del movimiento. Un hecho novedoso en estos años es la aparición de figuras independientes en el marco del movimiento, personas sin filiación política conocida y que resultan menos manipulables ${ }^{28}$. Los militantes de las organizaciones tienen un perfil cada vez más profesional, con conocimiento y uso de un lenguaje técnico que amplía las posibilidades de crítica bajo la apariencia de acciones alejadas de la valoración política, cuando en realidad arremeten directamente contra la propia esencia del sistema ${ }^{29}$.

El discurso oficial, a través de su compromiso con la causa de los derechos humanos convierte este campo en una plataforma privilegiada de acción política. Por éste y otros motivos, los partidos contemplarán las organizaciones como instrumentos ideales para hacer política "por otros medios" a través de una división del trabajo en la cual las asociaciones pueden sobrepasar unos límites no permitidos a las fuerzas políticas.

La emergencia del movimiento de derechos humanos resultará decisiva en la transformación de la actitud de las autoridades hacia el tema. Los regímenes marroquí y tunecino pretenderán implicar a los diferentes partidos y orga-

27. Otras organizaciones que actúan en la tegión son, en Marruecos, el Comité de Defensa de los Derechos Humanos (CDDH), una escisión de la sección de Marrakech de la AMDH producida a principios de 1992. En Túnez, aún bajo Burguiba, se crea en mayo de 1987 la Asociación para la Defensa de los Derechos Humanos y las Libertades Públicas, dirigida por el antiguo ministro del Interior Dahoui Hannablia, con posturas oficialistas. Igualmente se constituye un Instinato Arabe de Derechos Humanos que aunque difícilmente se puede considerar una organización de derechos humanos lieva a cabo algunas iniciativas interesantes. En febrero de 1993 dieciocho personalidades tunecinas crean el Comité Nacional de Defensa de los Prisioneros de Opinión (CNDPO), que tiene como presidente a Salah Hamzaoui. Amnistía Internacional creó su primera sección en un país átabe en Túnez y está en vfas de abrir otra en Marruecos.

28. El estudio de las organizaciones de derechos humanos proveen de un magnífico indicador de la vitalidad de la sociedad civil, relacionada con el desarrollo de un espacio de libertades para el conjunto de la sociedad en la cual el individuo se convierte en ciudadano-militante. La sociedad civil en el conjunto del Magreb se encuentra todavfa en un estadio embrionarto (El Aouf1, 1992), por lo que a nivel de derechos humanos la concenciación sobre la materia se encuentra relegada a una élite muy determinada pero en crecimiento paulatino.

29. Referencia continuada a figuras jurfdicas y a articulos de la Constitucion, a las leyes marroquíes o a textos y pactos internacionales de derechos humanos ratificados por los respectivos paises. 
nizaciones en las iniciativas y reformas a implantar. Gracias a su aceptación de participar en la burocracia de derechos humanos - aunque condicionada y no exenta de crítica a su actuación- las diferentes organizaciones y grupos legitiman la acción oficial y muestran su confianza en la posibilidad de un cambio-evolución que emane del interior del propio sistema ${ }^{30}$.

Las acciones iniciadas, y en general todo el proceso de institucionalizacion, se convierten en escudos que se interponen entre las denuncias de transgresión de la legalidad y la responsabilidad de los dirigentes de unos Estados con poderes altamente centralizados. El desconcierto de las organizaciones de derechos humanos frente a los carnbios es grande; como afirma la LTDH: «En otros tiempos las cosas eran completamente claras. Existían violaciones masivas de los derechos humanos y nosotros estábamos al otro lado de la barrera. Ahora nuestras relaciones son más complejas con el nuevo poder. Porque se trata de un régimen que, a nivel de discurso, reconoce los valores de los derechos humanos" (Marzouki, 1990: 16). En el caso de los organismos de derechos humanos, éstos han de ser los que vigilen el respeto de las leyes, pongan en evidencia las deficiencias de la situación actual y propongan las reformas a realizar. A traves de estas funciones se convierten en co-responsables de la gestión del campo de los derechos humanos, aunque sus atribuciones reales imposibiliten que en la práctica puedan ejercer este papel. Su supeditación en todos los casos a las decisiones emanadas desde la cúspide del poder convierte esta responsabilidad en mero artificio discursivo.

El significativo desarrollo de una oposición más coordinada y la emergencia del movimiento de derechos humanos ha coincidido con una serie de factores que pueden haber contribuido a replantear la relación con estos grupos y a iniciar la apropiación de la temática de los derechos humanos por los regímenes en el poder. Nos referimos a la percepción de una serie de "amenazas" que aconsejan la formación de nuevos consensos y habrían impulsado al otorgamiento de ciertos gestos hacia la oposición. Sin duda uno de los factores percibidos como más desafiadores por los dos países magrebies es el auge de los movimientos islamistas. En Túnez - - país en el cual durante el período Burguiba se había optado por una clara laicización de la sociedad, parcialmente corregida por Ben Alí a través de una política de símbolos-, el movimiento se encuentra especialmente desarrollado a nivel doctrinal y organizativo. En el caso de Marruecos, la legitimidad religiosa que ostenta Hasán II como

30. Recordamos como tanto en el CCDH marroquí como en el Comité Superior tunecino, se integran diferentes partidos políticos y asociaciones de derechos humanos. El ex-ministro de Derechos Humanos marroquf, Omar Azziman, habla sido presidente de la Organización Marroquí de Derechos Humanos (OMDH). En el caso tunecino la tendencia a la cooptación es mucho más acusada, con la amplia participación de miembros de la Liga Tunecina de Derechos Humanos (LTDH) en el gobierno: E. Zmerli, presidente de la LTDH, es nombrado ministro de Salud en julio de 1988; otro presidente, Muhammad Charfi, ministro de Educación Nacional; el militante de la Liga, Sadok Chaabane, Ministro de Justicia y dos secretatios generales se integran igualmente en el gobierno (Larif-Beatrix, 1990: 776 y Lawyers Committee, 1993: 53). 
dirigente religioso (Emir al-Muminin) descendiente directo del profeta Mahoma, unido a una hábil manipulación del campo político-religioso y a una tepresión de los elementos islamistas más radicales (al igual que en Túnez los grupos islamistas no han podido constituirse en partidos), parece haber alejado por el momento el peligro de insurrección, aunque la amenaza de su reaparición se muestre periódicamente ${ }^{31}$. La percepción de la amenaza en la región se ve aumentada por el seguimiento de los acontecimientos que azotan la vecina Argelia y el miedo a un efecto emulación. Sin duda uno de los frenos posibles más efectivos al avance de estas fuerzas es el permitir la presencia de una oposición que compita sobre el terreno frente a los islamistas por la monopolización del "campo de antagonismo al régimen".

Un segundo elemento extrapolable al conjunto de la región es la delicada coyuntura económica que igualmente exige la concertación con los principales actores políticos. La gestión de los sacrificios impuestos en aras de la recuperación económica y el control de las explosiones periódicas de descontento de la población más paupérrima, pueden llegar a ser un incentivo a la ampliación de los sectores que participan en las decisiones político-económicas.

En el caso de Marruecos, el previsible final al largo conflicto del Sahara Occidental se plantea igualmente como una problemática que requiere una gestión delicada ${ }^{32}$. La cuestión ha aglutinado a las diferentes fuerzas políticas a través de la exacerbación de los sentimientos más nacionalistas; su desenlace, sea cual sea su signo, puede comportar el final definitivo de un consenso ya altamente resquebrajado, la desaparición de un componente decisivo del discurso unanimista y entrañar la vuelta a la escena marroquí de las fuerzas castrenses relegadas a los confines meridionales del Reino.

La observación de los últimos acontecimientos en la región muestra como estas "amenazas" no han sido consideradas lo suficientemente desafiadoras como para implicar un cambio de fondo. En ambos países se ha producido un bloqueo a la alternancia política y al reparto de poder, mientras que ha continuado la represión política. Ahora bien, estos factores sí han sido considerados lo bastante amenazantes como para exigir la concesión de gestos conciliadores hacia sectores especialmente activos.

\section{La acción internacional en defensa de los derechos humanos}

Un nuevo elemento que muestra la apropiación de la temática de los derechos humanos por los regímenes marroquí y tunecino es la adhesión formal a los

31. Piénsese, por ejemplo, en Fes en el atentado de finales de verano de 1994 en Fes, que causó la muere de dos turistas españoles. Sobre los movimientos islamistas en Marnuecos y Túnez, véanse Lamchichi (1989) y Burgat y Dowell (1993).

32. El 30 de agosto de 1988 Marruecos y el Frente Polisario aceptan en Ginebra la propuesta del secretario general de las Naciones Unidas sobre la modalidad de referéndum. Uno de los principales obstáculos a su celebración, que ba sido pospuesta en diferentes ocasiones, en la discrepancia entre las partes respecto a la determinación del cuerpo de votantes. 
principales instrumentos internacionales de defensa de los derechos humanos. Ambos países han firmado y ratificado durante los últimos años prácticamente todos los pactos y textos de las Naciones Unidas relativos a los derechos humanos ${ }^{33}$, han presentado informes sobre la situación de determinados derechos a los comités pertinentes y se encuentran representados de manera activa en la mayor parte de órganos de las NN.UU. que se ocupan de la cuestión ${ }^{34}$. La voluntad de legitimación internacional resulta evidente, lo que nos hace plantear la existencia de presiones efectuadas desde el sistema internacional sobre el respeto de los derechos humanos a rivel interno.

En términos generales las iniciativas internacionales de promoción de los derechos humanos no han constituido una acción organizada ni de alta intensidad, sin que por eilo se hayan de menospreciar sus posibles efectos. La temática de los derechos humanos ha provocado "distorsiones" y situaciones delicadas que enturbian las relaciones exteriores de estos países magrebíes.

Entre las organizaciones internacionales no gubernamentales con una acción significativa en la zona hay que destacar la labor realizada por Amnistfa Internacional (AI), que ha dedicado un considerable esfuerzo a la difusión de información sobre los dos países a través de la publicación de numerosos comunicados y ha enviado misiones de investigación sobre el terreno en diversas ocasiones. Las relaciones de la organización han sido especialmente conflictivas con las autoridades marroquíes desde finales de los ochenta, cuando se intensifica la denuncia de lias violaciones cometidas en este país. El duro intercambio de informes, conträinformes, acusaciones y desmentidos lleva en diversas ocasiones al monarca alauí a atacar públicamente a Amnistía ("Yo he lanzado una invitación a $\mathrm{AI}$ a través de la prensa anteayer diciendo que estoy preparado para que ellos vengan, para constatar que son unos mentirosos" ${ }^{35}$ ) e incluso a expulsar de su territorio a miembros de la organización. En cuanto a Túnez, los comunicados de AI serán especialmente duros desde principios de la década de los noventa y comportarán igualmente relaciones tensas con el gobierno tunecino.

Los diferentes órganos de lás Naciones Unidas competentes en materia de derechos humanos han realizado una interesante labor de escrutinio y vigilancia de la cuestión, dedicando varias de sus sesiones a la revisión de la siruación de los derechos humanos y a la discusión de los informes presentados por estos países.

33. El Pacto Internacional de Derechos Civiles y Polfticos, el Pacto Internacional de Derechos Económicos, Sociales y Cułturales o Convención contra la Tortura y Otros Tratos o Penas Crueles, Inhumanos o Degradances, entre otros.

34. Túnez ha tenido un papel motot en la Comisión Africana de derechos humanos y de los pueblos, dependiente de la OUA, y es tuno de los primeros países africanos que somete un informe a este organismo. Marruecos se retiró de la organización en 1984 como protesta por el ingreso de la RASD.

35. Entrevista a Hasán II realizada por periodistas franceses el 26 de noviembre de 1985 (Nouvel Observateur). 
A nivel bilateral son sin duda los Estados Unidos y Francia los países que por sus vínculos históricos y sus estrechas relaciones económicas, financieras y militares pueden liegar a ejercer una mayor influencia sobre Marruecos y Túnez. Aquellos países han afirmado explícitamente su compromiso con el fomento los derechos humanos en el mundo a través de su política exterior, no solamente en los fórums multilaterales, sinó también a través de sus telaciones bilaterales. A pesar de este compromiso, las presiones sobre los gobiernos marroquí y tunecino se han limitado a intervenciones no públicas a través de los canales de la diplomacia secreta y preferenremente teniendo como matco los contactos regulares entre los respectivos dirigentes. Su objetivo ha sido muy limitado, reduciéndose a actuaciones en relación con dossiers muy concretos que mayoritariamente implicaban, directa o indirectarnente, a nacionales franceses o estadounidenses o a casos que habían recibido un trato preferente por los medios de comunicación respectivos ${ }^{36}$. A pesar de la escasa implicación de los gobiernos occidentales durante la década de los ochenta hasta la actualidad, la temática de los derechos humanos ha interferido en el transcurso cotidiano de las relaciones, especialmente con Francia, país que conserva estrechos lazos con el Magreb. En relación con el activismo en defensa de los derechos humanos hay que recordar que en suelo francés habita una importante comunidad de marroques y tunecinos, en su mayor parte inmigrantes en busca de mejores condiciones de vida, pero también un sector de exiliados políticos especialmente activo. Esto sin duda ha influido en la proliferación de organizaciones que se octupan de la defensa de los derechos humanos desde territorio francés ${ }^{37}$.

La temática de los derechos humanos ha estado muy presente en las relaciones franco-marroquíes ${ }^{38}$. Cada viaje de Mitterrand y de otras personalidades francesas a Marruecos coincidirá con el conocimiento de un nuevo dossier relativo a los derechos humanos, con el consiguiente embarazo oficial y el malestar de una parte de la opinión pública francesa y, especialmente, del pro-

36. Citamos como ejemplos las presiones de EE.UU. para que se libere al militar M'barek Tuil, implicado en el golpe de estado de 1972 y casado con una ciudadana estadounidense, mientras que personalidades políticas francesas se habrían interesado por el caso de Abraham Serfary, dirigente marxista-leninista de origen judio.

37. Destacamos, entre otras, la Asociación de defensa de los Derechos Humanos en Marruecos (ASDHOM) o el movimiento Túnez Democracia Ahora. Otros grupos integrados exclusivamente por franceses han ejercido una constante acción de cabildeo frente a las autoridades francesas, como es el caso de los Comités de Lucha contra la Represión en Marruecos (CLCRM).

38. Publicación del libro de Gilles Perrault (1991) Nuestro amigo el Rey, que provoca la anulación por parte de Marruecos de las numerosas actividades previstas para la conmemoración del "Tiempo de Marruecos" en Francia y el envío por parte de miles de marroquíes de cartas de protesta al presidente $F$. Mitrerrand, curiosamente muchas de ellas con las mismas faltas de ortografta. Otto asunto a destacar es la polémica alrededor de la expulsión por parte de Francia del oponente marroqui Mumen Diuri en 1991, que es anulada por una sentencia del Tribunal Administrativo de París cuando Diuri ya se enctzentra en Gabón. 
pio PS francés. La situación llega al extremo de que Hasán II descalificará públicamente a la esposa del presidente, Danielle Mitterrand, por sus campañas en defensa de los derechos humanos en Marruecos y a favor de la causa saharaui. Igualmente, y tanto en relación con Marruecos como con Túnez, cada desplazamiento a Francia de una autoridad procedente de alguno de estos países será aprovechada para convocar manifestaciones de protesta y para plantear delicadas preguntas por parte de los petiodistas occidentales respecto a los dossiers de derechos humanos más delicados.

Las relaciones con Estados Unidos serán menos problemáticas. A finales de los años ochenta se observa un ligero cambio bajo la administración Bush, cuando los informes que el Departamento de Estado viene elaborando desde 1974 sobre el estado de los derechos humanos en el mundo empiezan a ser especialmente críticos y provocan las protestas de las embajadas marroquí y tunecina. Igualmente, en los debates del Congreso la cuestión, prácticamente ignorada en períodos anteriores, es planteada de manera insistente ${ }^{39}$.

La respuesta oficial matroquí i tunecina a los ataques procedentes del exterior busca exacerbar los sentimientos nacionalistas a través de la afirmación de que aquellos responden a campañas orquestadas por potencias enemigas que desean mancillar la reputación del país. Ben Alí declara: «[...] la propagación de las informaciones sobre la tortura es un atentado contra la reputación de Túnez" (Daoud, 1993: 947); mientras Hasán II afirma que: "Amnistía Internacional y otros quieren ejercer sobre nosotros su control, como si nosotros estuviésemos aún bajo el F'rotectoradon (Hassan II, 1991: 46).

Un elemento que resulta esencial para evaluar la posible influencia de la crítica desde el exterior son los importantísimos lazos económicos y financiero de Túnez y Marruecos con ciertos países occidentales y con organismos financieros internacionales. Las economías marroquí y tunecina se caracterizan por su extraversión económica, que las convierte en altamente vulnerables frente a los constreñimientos del sistema económico internacional ${ }^{40}$. En los dos casos, marroguí y tunecino, el recurso a la financiación exterior suple las tan temidas reformas estructurales del sistema, que podrian provocar oleadas de descontento entre la población más depauperada y obligarían a profundizar el diálogo con la oposición en busca de apoyo a las opciones económicas (la otra alternativa sería el recurso a la represión generalizada) (Leveau, 1987).

Sin duda existe el temor a lés posibles consecuencias económicas de las críticas exteriores sobre la situación de los derechos humanos (como podria ser

39. Véase, por ejemplo, Estados Unirios (1991).

40. Citamos únicamente algunos de los puntos que muestran el anclaje en el interior del sistema economico internacional; las dos economías magrebies han continuando las opciones del perfodo colonial; los intercambios comerciales, de naturaleza muy asimétrica, convierten estos paises en altamente dependientes de sus exportaciones a Europa y, principalmente, a Francia, el recurso a los préstamos bilaterales o procedentes del Fondo Monerario Internaciona (FMI) y del Banco Mundial (BM) se ha acelerado en los años ochenta, y la dependencia de la llegada de divisas procedentes de las remesas de los trabajadores inmigrantes es muy elevada. Sobre estas cuestiones véase Mir (1993). 
la retirada de confranza de los organismos internacionales o las reticencias de los inversores a actuar en los dos países) ${ }^{41}$. El propio primer ministro marroquí Azzedin Laraki, lo reconoce: "No se puede permanecer perplejo frente a esta parcialidad y frente a la campaña contra Marruecos en un momento en el cual tiende legítimamente, como todos los países despedazados por la colonización, a conseguir su integridad y en el momento en el cual realiza un despegue económico prometedor y suscita el interés de los inversores extranjeros $\$ 2$.

Claramente, los cambios formales identificados también van dirigidos hacia una mejora de la imagen exterior. En este sentido se ha de interpretar tanto el discurso oficial como, y sobretodo, la creación de una burocracia de derechos humanos que "nacionalice" la temática y convierta en superflua toda ingerencia externa.

\section{Evolución o revolución en el campo de los derechos humanos}

Los cambios seńalados en los primeros apartados no se han traducido en una revolución en el campo de los derechos humanos. En primer lugar, las mutaciones observadas no se han realizado en base al diálogo, sino como concesiones otorgadas desde la cúspide del poder. Aún menos las novedades de los años noventa han comportado un cambio en la naturaleza de los sistemas ni en el reparto de poder. En ambos países se ha bloqueado el acceso de la oposición al gobierno a través del trucaje de los comicios y, en el caso marroquí, por la negativa real a aceptar las condiciones de la oposición a su integración. Si la vía del cambio no ha sido la escogida para dar respuesta a las crecientes presiones sobre el régimen, la opción alternativa ha de ser, bien la regulación de la escena política a través de la represion, bien el intento de otorgar una apariencia de cambio al inmobilismo. En relación a esto último, la temática de los derechos humanos se ha mostrado como un excelente instrumento político en manos de las autoridades martoquíes y tunecinas, que han deseado amortiguar las críticas realizadas desde el interior y desde el exterior en un momento en el cual la problemática ha cobrado un especial protagonismo. Una última reflexión se impone respecto al futuro y a las consecuencias de dicha apropiación. El discurso oficial acompañado de la edificación de una serie de instituciones que velen por la temática de los derechos humanos provoca indirectamente una dinámica de legitimación de la cuestión. Las repetidas alusiones otorgan fuerza al combate por el respeto de los derechos humanos y legitiman la acción de las organizaciones. La temática de los derechos humanos

41. Muestra de la incidencia de la temática en cuestiones económicas es el bloqueo por el Parlamento Europeo el 15 de enero de 1992 del cuarto protocolo financiero con Marruecos por un valor total de 600 millones de dólares debido a la actitud del régimen respecto a los derechos humanos y hacia el referéndum que se ha de celebrar en el Sahara Occidental. Aunque la postura no es secundada por la Comisión, que plantea como alternativa una zona de libre cambio con Marruecos, se trata de una clara advertencia para el Reino alaú

42. L'Opinion, 22/2/1990. 
queda enraizada en el interior del campo político-social. Si esto no produce un cambio en el estado de los derechos humanos a corto plazo, sí puede, en cambio, llegar a impulsar transformaciones profundas en el futuro, relacionadas con una mayor concienciación de la sociedad civil.

Hasta el momento, la tan esperada revolución en el campo de los derechos se habrá limitado -especialmente en el caso tunecino y con algunas reservas respecto a Marruecos - a una operación cosmética y de maquillaje que no sabrá esconder los estragos calısados por unas prácticas que, a la manera del retrato de Dorian Gray, se enctientran cubiertas por un velo. El movimiento de derechos humanos pretenderá sustituir el espejo de los engaños y artificios institucionales por el implacable lienzo.

\section{Bibliografia}

Al-AHNAF, Mustapha (1993). "Maroc: force et faiblesses des acteurs juridiques». Maghreb-Machrek, núm. 142, p. 16-23.

AMNISTIA INTERNACIONAL (1994). Tunisie: du discours à la réalité. París: Éditions Francophones d'Amnesty International.

BASRI, D.; ROUSSET, M.; VEDEL, G. (dirs.) (1994). Le Maroc et les droits de l'homme: Positions, réalisations et perspectives. Paris: L'Harmattan.

Bennouna, Mehdi (1993a). "Le mouvement des droits de l'homme au Maroc: du sujet au citoyen\%. Memoria de doctorado presentada al IEP de París.

- (1993b). "Les mouvements des droits de l'homme au Maroc en quête de citoyenneté». Hommes Ĺ Libertés, núm. 70, p. 48-51.

BESSIS, Sophie (1989). «Banque nondiale et FMI en Tunisie: une évolution sur trente ans". Annuaire de l'Afrique du Nord 1987. París: CNRS, p. 135-148.

BURGAT, François; DOWELl, William (1993). The Islamic movement in North Africa. Austin, Texas: Center for Middle Eastern Studies.

CAMAU, Michel (1971). La notion de démocratie dans la pensée des dirigeants magbrébins. Paris: CNRS.

DaOUD, Zakia (1991). "Chronique Tunisie». Annuaire de l'Afrigue du Nord 1989. París: CNRS, p. 679-702.

- (1993). "Chronique Tunisie". Annuaire de l'Afrique du Nord 1991. París: CNRS, p. $941-968$.

DONNELly, Jack (1986). "International Human Rights: a Regime Analysis", International Organization, vol. 40, núm. 3, p. 599-643.

- (1989). Universal Hhuman Rights in Theory and Practice. Nueva York; Ithaca: Cornell University Press.

DRISS, Rachid (1993). "Démocratie et droits de l'homme en Tunisie", en MarTIN MuNoz, Gema (ed.) Democracia y derechos humanos en el mundo árabe. Madrid: Ministerio de Asuntos Exteriores: AECI: ICEM, p. 149-162.

EL AOUfi, Noureddine (dir.) (1992). La societé civile au Maroc: Approches. Casablanca: SMER.

ESTADOS Unidos. Cámara de Kepresentantes. Comité de Relaciones Exteriores. Subcomité de Derechos Humanos y Organizaciones Internacionales (1991). Human Rights in the Maghreb and Mauritania. Washington, DC.: US Government Printing Office. 
FORSYTHE, David P. (1990). «Human rights in US Foreign Policy: retrospect and prospectn. Political Science Quarterly, vol. 105, ním. 3, p. 435-454.

Hassan II $(1991,1992$, 1993). Discours et interviews de Sa Majesté le Roi Hassan $I I$. Rabat: Ministère de l'Information.

KAREM, Mohamed (1991). "La notion des droits de l'homme aut Maghreb: Essai sur une nouvelle culture politiquen. Tesis de doctorado en Ciencia Política. Facultar de Derecho y de Ciencia Política d'Aix-Marseiłle.

- (1993). "La question des droits de l'homme au Maghreb", en MARTIN MUÑOZ, Gema (ed.) Democracia y derechos bumanos en el mundo arabe. Madrid: Ministerio de Asuntos Exteriores: AECI: ICEM, p. 125-I48.

LAMCHICHI, Abderrahim (1989). Islam et contestation au Maghreb. París: L'Harmattan.

LARIF-BEATRX, Asma (1989). "Chronique Tunisienne". Annuaire de l'Afrique du Nord 1987. París: CNRS, p. 751-812.

- (1990). "Chronique Tunisienne". Annuaire de l'Afrique du Nord 1988. París: CNRS, p. 772-787.

LARIF-BEATRX, Asma (1991). "Changement dans la symbolique du pouvoir en Tunisie". Annuaire de l'Afrique du Nord 1989. París: CNRS, p. 141-151.

Lawyers COMMitTeE For Human Rights (1993). Promise Unfulfilled: Human Rights in Tunisia since 1987. Nueva York: Lawyers Committee.

LEVEAU, Rémy (1987). "Stabilité du pouvoir monarchique et financement de la dette». Maghreb-Machrek, núm. 118, p. 5-19.

- (1993). Le sabre et le turban: l'avenir du Maghreb. París: François Bourin.

MARTIN MUNoz, Gema (ed.) (1993). Democracia y derechos humanos en el mundo árabe. Madrid: Ministerio de Asuntos Exteriores: AECI: ICEM.

MARZOUKI, Moncef (1990). "L'an III de l'ère des droits de l'homme». Le Maghreb, núm. 224, p. 10-17.

MrR, Pere (1993). Economia $i$ sector exterior en els estats de la Unío del Magrib Arab (anys 70 i 80). Bellaterra: Universitat Autònoma de Barcelona. Centre d'Estudis sobre la Pau i el Desarmament. 2 vol.

PerRault, Gilles (1991). Nuestro amigo el Rey. Barcelona: Plaza \& Janés. Cambio 16.

VINCENT, R.J. (1986a). Foreign Policy and Human Rights. Cambridge: Cambridge University Press.

- (1986b). Human Rights and International Relations. Cambridge: Cambridge University Press.

WaLTZ, Susan (1991). "Making Waves: the Political Impact of Human Rights Groups in North African. The Journal of Modern African Srudies, vol. 29, núm. 3, p. $481-504$.

WARE, L.B. (1988), "Ben Ali's Constitutional Coup in Tunisia". The Middle East Joumal vol. 42, núm. 4, p. 587-601. 